\title{
Quantifying non-Newtonian effects in rotating boundary-layer flows
}

\author{
P.T. Griffiths ${ }^{\text {a,*, S.J. Garrett }}{ }^{\text {b }}$, S.O. Stephen ${ }^{\mathrm{c}}$, Z. Hussain ${ }^{\mathrm{d}}$ \\ a Department of Mechanical Engineering and Mathematical Sciences, Oxford Brookes University, Oxford, United Kingdom \\ ${ }^{\mathrm{b}}$ Department of Engineering, University of Leicester, Leicester, United Kingdom \\ ' School of Mathematics and Statistics, University of Sydney, Sydney, NSW 2006, Australia \\ d School of Computing, Mathematics and Digital Technology, Manchester Metropolitan University, United Kingdom
}

\section{A R T I C L E I N F O}

\section{Article history:}

Available online $\mathrm{xxxx}$

\section{Keywords:}

Rotating boundary layers

Non-Newtonian

Convective instability

\section{A B S T R A C T}

The stability of the boundary-layer on a rotating disk is considered for
fluids that adhere to a non-Newtonian governing viscosity relationship. For
fluids with shear-rate dependent viscosity the base flow is no longer an
exact solution of the Navier-Stokes equations, however, in the limit of large
Reynolds number the flow inside the three-dimensional boundary-layer can
be determined via a similarity solution.
The convective instabilities associated with flows of this nature are
described both asymptotically and numerically via separate linear stability
analyses. Akin to previous Newtonian studies it is found that there exists two
primary modes of instability; the upper-branch type I modes, and the lower-
branch type II modes. Results show that both these modes can be stabilised
or destabilised depending on the choice of non-Newtonian viscosity model. A
number of comments are made regarding the suitability of some of the more
well-known non-Newtonian constitutive relationships within the context of
the rotating disk model. Such a study is presented with a view to suggesting
potential control mechanisms for flows that are practically relevant to the
turbo-machinery industry.

(C) 2016 Elsevier Masson SAS. All rights reserved.

\section{Introduction}

The stability and transition of the boundary-layer on a rotating disk is a classical fluid mechanics problem that has attracted a great deal of attention from numerous authors over many decades. The first theoretical investigation of this three-dimensional problem was performed by von Kármán [1]. He showed that the steady incompressible flow induced by the rotation of an infinite plane with uniform angular velocity is an exact solution of the Navier-Stokes equations.

The boundary-layer flow is characterised by the lack of a radial pressure gradient near to the disk to balance the centrifugal forces so the fluid spirals outwards. The disk acts as a centrifugal fan, the fluid emanating from the disk being replaced by an axial flow directed back towards the surface.

\footnotetext{
* Corresponding author.

E-mail address: pgriffiths@brookes.ac.uk (P.T. Griffiths).
}

Batchelor [2] showed that this type of flow is in fact just a limiting case of a whole number of flows with similarity solutions in which both the infinite plane and the fluid at infinity rotate with differing angular velocities. The corresponding limiting case, when the infinite plane is stationary and the fluid at infinity rotates at a constant angular velocity, was first described by Bödewadt [3].

A vast wealth of material exists concerning the solutions of the Newtonian rotating disk equations; the interested reader is referred to Zandbergen and Dijkstra [4]. The authors provide a thorough review of the major contributions made postdating von Kármán's seminal work.

The pioneering study of Gregory et al. [5] contains the first observation of the stationary cross-flow vortices on a rotating disk. The flow is convectively unstable, within certain regions, to disturbances stationary in the frame rotating with the disk. These disturbances are excited by roughnesses on the surface and, because these roughnesses are fixed in time in the rotating frame, the stationary disturbances are consistently excited and reinforced such that they are evident in flow-visualisation experiments. 
Theoretical studies of convective modes within the rotatingdisk system have shown that the flow is susceptible to two distinct modes of instability. The type I (upper-branch) mode due to the cross-flow instability and the type II (lower-branch) mode attributed to external streamline curvature. Because of this the neutral curve has a characteristic two-lobed structure, as noted by Malik [6] and Lingwood [7], amongst others. Hall [8] demonstrated that in the limit of large Reynolds number the upper and lower branches of the neutral curve can be described (with excellent agreement) using asymptotic theory. More recently, the aforementioned pioneering studies have been extended to include rotating spheres, see Garrett and Peake [9] and rotating cones, see Garrett et al. [10] and Hussain et al. [11]. In all cases the flow can be stabilised (or destabilised) with control of the governing parameter, that being the spin rate, in the case of the sphere, and the half-angle, in the case of the cone. Indeed, research on the von Kármán boundary-layer remains a topic of active study, the interested reader is referred to the recent review article by Lingwood and Alfredsson [12].

Rotating disk flows have practical relevance to turbo-machinery where non-Newtonian fluids are commonplace. However, far less attention has been given to the corresponding non-Newtonian rotating disk problem. Very recently Griffiths [13] determined the base flow profiles for numerous generalised Newtonian fluid models. Using these results we investigate the convective instability of the boundary-layer on a rotating disk for shear-thinning fluids that adhere to the power-law and Carreau constitutive governing viscosity relationships. Results show that accurately modelling the variation of viscosity within the boundary-layer is of paramount importance.

\section{Formulation}

We consider the flow of a steady incompressible nonNewtonian fluid due to an infinite rotating plane located at $z^{*}=0$ (here the superscript $*$ denotes a dimensional quantity). The plane rotates about the $z^{*}$-axis with angular velocity $\Omega^{*}$. The motion of the fluid is in the positive $z^{*}$ direction, the fluid is infinite in extent and the only boundary is located at $z^{*}=0$. In a rotating frame of reference the continuity and Cauchy momentum equations are expressed as

$$
\begin{aligned}
& \nabla^{*} \cdot \boldsymbol{u}^{*}=0 \\
& \frac{\partial \boldsymbol{u}^{*}}{\partial t^{*}}+\boldsymbol{u}^{*} \cdot \nabla^{*} \boldsymbol{u}^{*}+\boldsymbol{\Omega}^{*} \times\left(\boldsymbol{\Omega}^{*} \times \boldsymbol{r}^{*}\right)+2 \boldsymbol{\Omega}^{*} \times \boldsymbol{u}^{*} \\
& =-\frac{1}{\rho^{*}} \nabla^{*} p^{*}+\frac{1}{\rho^{*}} \nabla^{*} \cdot \boldsymbol{\tau}^{*} .
\end{aligned}
$$

Here $\boldsymbol{u}^{*}=\left(\tilde{U}^{*}, \tilde{V}^{*}, \tilde{W}^{*}\right)$ are the velocity components in cylindrical polar coordinates $\left(r^{*}, \theta, z^{*}\right), t^{*}$ is time, $\Omega^{*}=\left(0,0, \Omega^{*}\right)$ and $\boldsymbol{r}^{*}=\left(r^{*}, 0, z^{*}\right)$. The fluid density is $\rho^{*}$ and $p^{*}$ is the fluid pressure. For generalised Newtonian models the stress tensor is given by $\boldsymbol{\tau}^{*}=\mu^{*}\left(\dot{\gamma}^{*}\right) \dot{\gamma}^{*}$, where $\dot{\gamma}^{*}=\nabla^{*} \boldsymbol{u}^{*}+\left(\nabla^{*} \boldsymbol{u}^{*}\right)^{\mathrm{T}}$ is the rate of strain tensor and $\mu^{*}\left(\dot{\gamma}^{*}\right)$ is the non-Newtonian viscosity. The magnitude of the rate of strain tensor is $\dot{\gamma}^{*}=\sqrt{\left(\dot{\gamma}^{*}: \dot{\gamma}^{*}\right) / 2}$. The governing relationships for $\mu^{*}\left(\dot{\gamma}^{*}\right)$ that will be considered herein are:

$\mu^{*}=m^{*}\left(\dot{\gamma}^{*}\right)^{n-1}$,

$\mu^{*}=\mu_{\infty}^{*}+\left(\mu_{0}^{*}-\mu_{\infty}^{*}\right)\left[1+\left(\lambda^{*} \dot{\gamma}^{*}\right)^{2}\right]^{(n-1) / 2}$,

these are the power-law and Carreau models, respectively. Here $m^{*}$ is the consistency coefficient and $n$ is the fluid index, for $n>1$ the fluid is said to be shear-thickening, whilst for $n<1$ the fluid is said to be shear-thinning. The Newtonian viscosity relationship is recovered when $n=1$ and $\mu_{0}^{*}=\mu_{\infty}^{*}$, respectively. The infinite-shear-rate viscosity is $\mu_{\infty}^{*}$, the zero-shear-rate viscosity is $\mu_{0}^{*}$ and $\lambda^{*}$ is the characteristic time constant, often referred to as the 'relaxation time'.

In the Newtonian limit an exact solution of (1) exists, as was first determined by von Kármán [1]. However, no such solution exists for flows with $n \neq 1$. It is only in the large Reynolds number limit that the leading order boundary-layer equations admit a similarity solution analogous to the exact Newtonian solution. The governing boundary-layer equations are given in Griffiths [13], for brevity we exclude these here.

We introduce the generalisation of the classic Newtonian similarity solution in order to solve for the steady mean flow relative to the disk. The dimensionless similarity variables are defined by

$$
\begin{aligned}
U(\eta) & =\frac{\tilde{U}_{0}^{*}}{r^{*} \Omega^{*}}, \quad V(\eta)=\frac{\tilde{V}_{0}^{*}}{r^{*} \Omega^{*}}, \\
W(\eta) & =\frac{\tilde{W}_{0}^{*}}{\chi^{*}}, \quad P(\eta)=\frac{\tilde{P}_{1}^{*}}{\rho^{*}\left(\chi^{*}\right)^{2}},
\end{aligned}
$$

where $\chi^{*}=\left[v^{*} /\left(r^{*}\right)^{1-q}\left(\Omega^{*}\right)^{1-2 q}\right]^{1 /(q+1)}$. Here $\left(\tilde{U}_{0}^{*}, \tilde{V}_{0}^{*}, \tilde{W}_{0}^{*}\right)$ are the leading order velocity components, $\tilde{P}_{1}^{*}$ is the leading order fluid pressure term and $v^{*}=\sigma^{*} / \rho^{*}$ is the kinematic viscosity. The dimensionless similarity coordinate is $\eta=r^{(1-q) /(q+1)} z$, where here $r$ and $z$ have been made dimensionless with respect to $L^{*}=$ $\left[v^{*} /\left(\Omega^{*}\right)^{2-q}\right]^{1 / 2}$. For "power-law" fluids $q=n$ and $\sigma^{*}=m^{*}$, whereas for "Carreau" fluids $q=1$ and $\sigma^{*}=\mu_{\infty}^{*}$.

Thus the laminar-flow profiles are determined from the following set of non-linear ordinary differential equations:

$$
\begin{aligned}
2 U+\bar{\eta} U^{\prime}+W^{\prime} & =0, \\
U^{2}-(V+1)^{2}+(W+\bar{\eta} U) U^{\prime}-\left(\mu U^{\prime}\right)^{\prime} & =0, \\
2 U(V+1)+(W+\bar{\eta} U) V^{\prime}-\left(\mu V^{\prime}\right)^{\prime} & =0,
\end{aligned}
$$

where $\bar{\eta}=\eta(1-q) /(q+1)$ and the primes denote differentiation with respect to $\eta$. The power-law viscosity function is given by

$\mu=\left[\left(U^{\prime}\right)^{2}+\left(V^{\prime}\right)^{2}\right]^{(n-1) / 2}$,

whilst the Carreau viscosity function takes the form

$\mu=1+c_{0}\left\{1+k^{2}\left[\left(U^{\prime}\right)^{2}+\left(V^{\prime}\right)^{2}\right]\right\}^{(n-1) / 2}$.

Here $c_{0}=\left(\sigma^{*}-\mu_{0}^{*}\right) / \sigma^{*}$ is the viscosity ratio, and $k=r^{*} \lambda^{*}$ $\Omega^{*}\left(\rho^{*} \Omega^{*} / \sigma^{*}\right)^{1 / 2}$ is the dimensionless equivalent of $\lambda^{*}$. Throughout this study we fix the values of $c_{0}$ and $k$ such that $c_{0}=1$ and $k=100$, this is consistent with previous investigations, see, for example, Dabrowski [14].

Using a fourth-order Runge-Kutta quadrature routine twinned with a Newton iteration scheme to determine the values of the unknowns $U^{\prime}(0)$ and $V^{\prime}(0)$ the set of ordinary differential equations are solved subject to

$U(\eta=0)=V(\eta=0)=W(\eta=0)=0$,
$U(\eta \rightarrow \infty) \rightarrow 0, \quad V(\eta \rightarrow \infty) \rightarrow-1$.

Although the formulation here is different the results represent an exact reproduction of those detailed by Griffiths [13]. As such, here, we do not graphically present the radial, azimuthal and axial velocity profiles and instead choose to discuss the differences between the base flow characteristics, and their significance in relation to the resulting linear stability calculations, in Section 4. We note that throughout this analysis the reference Newtonian viscosity for the Carreau fluid problem is $\mu=1+c_{0} \neq 1$. 


\section{Stability analyses}

\subsection{Type I asymptotic analysis}

This analysis is based on the assumption that the Reynolds number is large and that the disturbances have wavelengths scaled on the boundary-layer thickness, $\delta=R e^{-1 /(q+1)}$, where

$\operatorname{Re}=\frac{\left(\Omega^{*}\right)^{2-q}\left(l^{*}\right)^{2}}{\nu^{*}}$,

is the asymptotic representation of the Reynolds number and $l$ is some reference length scale.

When considering the type I modes we observe the existence of three distinct layers. An inviscid layer, or zone, a wall layer and a critical layer. The inviscid zone encompasses the entirety of the boundary-layer, the wall layer is needed to ensure the no-slip condition at the wall is satisfied and the critical layer exists so that the singularities that arise within the inviscid zone are smoothed out.

The full details regarding the determination of the type I "power-law" modes has already been reported by Griffiths et al. [15]. For brevity we choose to omit the full details concerning the corresponding Carreau modes as the analysis is largely similar. Here we present only the most pertinent results. However, the interested reader is referred to Griffiths [16] for full details regarding the asymptotic structure of the type I modes for both the power-law and Carreau shear-thinning models.

Perturbing the self-similar boundary-layer flow, and neglecting non-linear terms, we arrive at a set of generalised Newtonian linear disturbance equations. These governing equations are dependent on both the unperturbed and perturbed viscosity functions. The perturbation of the viscosity owes from the first-order crossproduct terms associated with the generalised binomial expansion of (2).

We introduce the small parameter $\epsilon=\delta^{1 / 3}$ and following Hall [8], we consider disturbances proportional to

$E=\exp \left\{\frac{\mathrm{i}}{\epsilon^{3}}\left[\int^{r} \alpha_{A}(r, \epsilon) \mathrm{d} r+\theta \beta_{A}(\epsilon)\right]\right\}$,

where here $r$ has been made dimensionless with respect $l$ and $\alpha_{A}$ and $\beta_{A}$ are the radial and azimuthal wavenumbers, respectively. We seek to find neutrally stable disturbances thus $\alpha_{A}, \beta_{A} \in$ $\mathbb{R}$. Within this asymptotic framework the wavenumbers are expanded in the following forms

$\alpha_{A}=\alpha_{0}+\epsilon \alpha_{1}+\cdots$,

$\beta_{A}=\beta_{0}+\epsilon \beta_{1}+\cdots$.

At leading order, $\mathcal{O}\left(\epsilon^{-3}\right)$, in the inviscid zone we determine that the disturbances must satisfy a modification of Rayleigh's equation. At the next order we determine a nonhomogeneous form of Rayleigh's equation that is required to match with our leading order solution in the wall layer. This wall layer solution is given in terms of the decaying Airy function. Despite the appearance of additional viscous terms in the leading order governing equations we find that analytic solutions are obtainable. Matching these solutions between the two layers produces the following linear eigenrelation

$$
\begin{aligned}
-\frac{9^{1 / 3}}{\varsigma \Gamma\left(\frac{1}{3}\right)}= & 2 r^{2(q-1) /(q+1)}\left(\alpha_{0} \alpha_{1}+\frac{\beta_{0} \beta_{1}}{r^{2}}\right) I_{1} \\
& +r\left(\frac{\alpha_{1}}{\beta_{0}}-\frac{\alpha_{0} \beta_{1}}{\beta_{0}^{2}}\right) I_{2},
\end{aligned}
$$

where $\varsigma=\left\{\mathrm{i}\left[\alpha_{0} U^{\prime}(0) r+\beta_{0} V^{\prime}(0)\right] / A\right\}^{1 / 3}$ and the constant $A$ is a function of the fluid index $n$ and the value of viscosity at the
Table 1

Numerical values for the constants that relate to the asymptotic expansions of the neutral wavenumber (6a), and wave angle (6b), for shear-thinning Carreau fluids.

\begin{tabular}{lllll}
\hline$n$ & $\gamma_{0}$ & $\gamma_{1}$ & $\lambda_{0}$ & $\lambda_{1}$ \\
\hline 0.25 & 1.154 & 9.16 & 4.182 & 16.63 \\
0.5 & 1.112 & 9.06 & 4.135 & 16.56 \\
0.75 & 1.004 & 8.57 & 4.126 & 17.34 \\
0.95 & 0.822 & 7.58 & 4.215 & 19.00 \\
\hline
\end{tabular}

wall $\mu(0)$. The exact form of this constant differs depending on the fluid model in question. The integrals $I_{1}$ and $I_{2}$ are defined and calculated in Griffiths [16] for a range of shear-thinning power-law and Carreau boundary-layer flows.

Solving this eigenrelation enables us to determine expressions for the asymptotic representation of the wavenumber $\gamma_{A}$, and wave angle $\phi_{A}$, of the disturbances as functions of $R=r^{2 /(q+1)} R e^{1 /(q+1)}$. Here $R$ is the numerical representation of the Reynolds number. Doing so enables us to make direct comparisons between our asymptotic and numerical solutions. We find that

$$
\begin{aligned}
\gamma_{A} & =r^{(q-1) /(q+1)} \sqrt{\alpha_{A}^{2}+\frac{\beta_{A}^{2}}{r^{2}}} \\
& =\gamma_{0}-\gamma_{1} R^{-1 / 3}+\cdots,
\end{aligned}
$$

and

$$
\begin{aligned}
\phi_{A} & =\operatorname{arccot}\left(\frac{\alpha_{A} r}{\beta_{A}}\right) \\
& =\operatorname{arccot}\left(\lambda_{0}+\lambda_{1} R^{-1 / 3}+\cdots\right) .
\end{aligned}
$$

Numerical values for the constants $\gamma_{i}$ and $\lambda_{i}$ for a range of shearthinning Carreau flows are displayed in Table 1 . The equivalent power-law results are presented in Griffiths et al. [15].

In this study we present only the type I asymptotic analysis with the viewpoint of validating our numerical results when the Reynolds number is large. Indeed, initial investigations have begun on the corresponding type II analysis, we hope to report on this investigation in due course. For more details regarding the type I analysis see Griffiths et al. [15] and Griffiths [16].

\subsection{Numerical analysis}

The stability analysis, applied at a specific radial location, involves imposing infinitesimally small disturbances on the steady mean flow, in the form of scaled normal-mode quantities

$(u, v, w, p)=(\hat{u}, \hat{v}, \hat{w}, \hat{p})(\eta ; \alpha, \beta, \omega ; R, n) e^{\mathrm{i}(\alpha r+\beta \theta-\omega t)}$.

The frequency of the disturbance in the rotating frame is $\omega$ (taken to be zero in this stationary study), the complex radial wavenumber is $\alpha=\alpha_{\mathrm{r}}+\alpha_{\mathrm{i}}$ and $\beta$ is the real azimuthal wavenumber.

After making an approximation akin to the parallel-flow assumption and a viscous assumption stemming from the asymptotic results, the stability equations may be written as sets of six first-order ODEs using transformed perturbing variables $\phi_{i}(\eta)$ with $i=1,2 \ldots 6$. The governing equations for shear-thinning fluids that adhere to the power-law viscosity model can be found in Griffiths et al. [17], the equivalent equations for fluids that adhere to the Carreau viscosity model can be easily inferred therein. In all that follows the eigenvalue problem defined by the stability equations is solved with the homogeneous boundary conditions

$$
\begin{aligned}
\phi_{i}=0 & \text { at } \eta=0, \\
\phi_{i} \rightarrow 0 & \text { as } \eta \rightarrow \infty,
\end{aligned}
$$

for all $i$. This eigenvalue problem is solved for certain combinations of values of $\alpha, \beta$ and $\omega$ at each Reynolds number, $R$, and for 

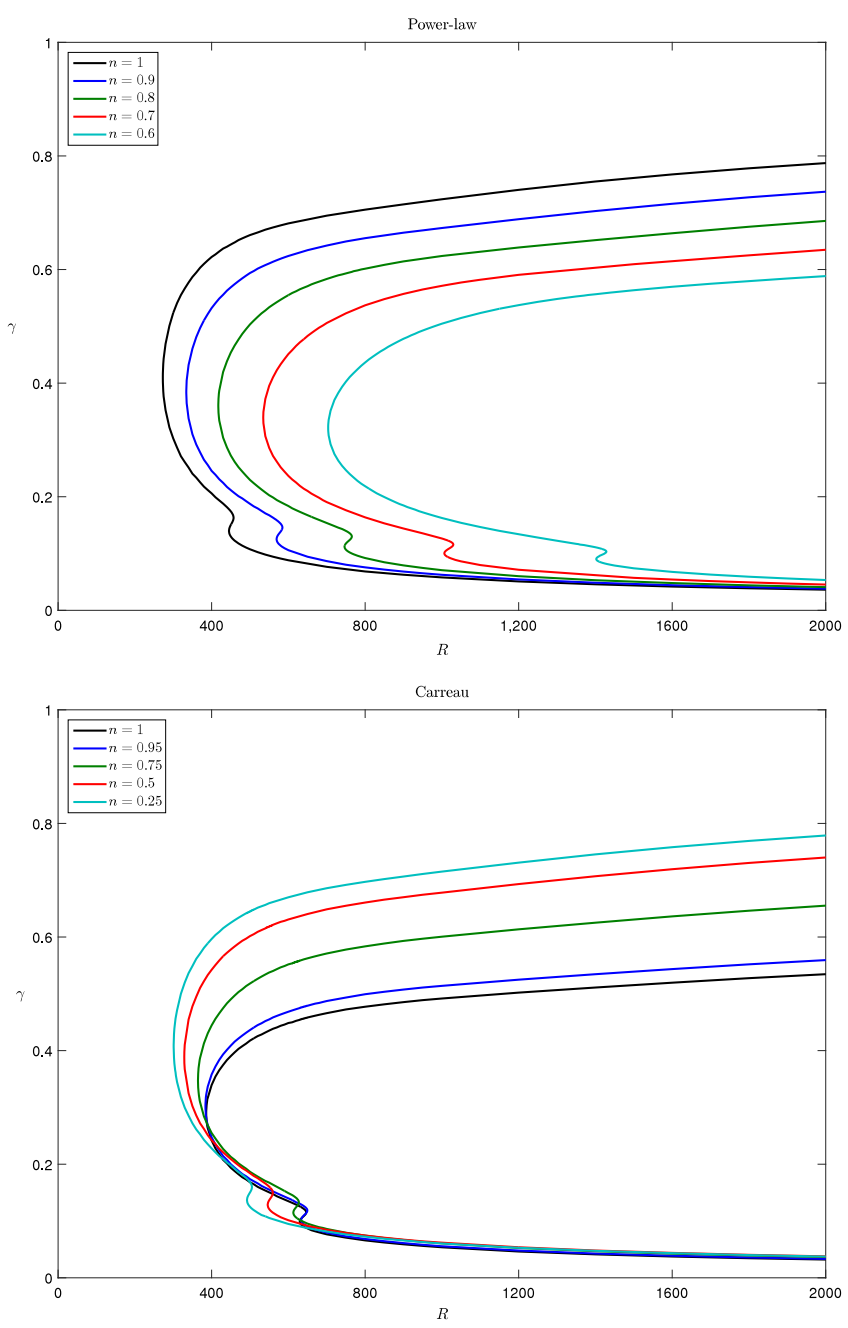

Fig. 1. Wavenumber neutral stability curves for shear-thinning power-law,and Carreau fluid flows.

the specified value of $n$. From these we form the dispersion relation, $D(\alpha, \beta, \omega ; R, n)=0$, at each $n$, with the aim of studying the convective instabilities. The step size in $\eta$ was reduced and the value of infinity increased until there were no discernible differences in the numerical results. The values taken were such that the boundary layer was approximated by 2000 equally spaced data points between $\eta=0$ and $\eta=20$. This discretisation is known to be consistent with Lingwood [7] and Garrett and Peake [18], for example, and represents an appropriate balance between accuracy and computational effort for each $n$.

In order to investigate the structure of the spatial branches at each $n$, we solve the dispersion relation for $\alpha$ whilst marching through values of $\beta$ at fixed $R$. For each $n$ in the particular range of interest two spatial branches determine the convective instability characteristics of the system. Neutral curves, defined by $\alpha_{\mathrm{i}}=0$, have been calculated for a variety of shear-thinning values of $n$ using both the power-law and Carreau constitutive viscosity laws.

All the results have been validated by equivalent calculations performed using a Chebyshev polynomial discretisation method. For details on the implementation of this method, for Newtonian rotating disk flows, the interested reader is referred to Alveroglu et al. [19]. Excellent agreement was found between the two numerical schemes. Some minor discrepancies were found in the values of the type II critical Reynolds numbers. However, as noted by Garrett et al. [20], the determination of this mode is strongly dependent on the choice of numerical solution method, whereas
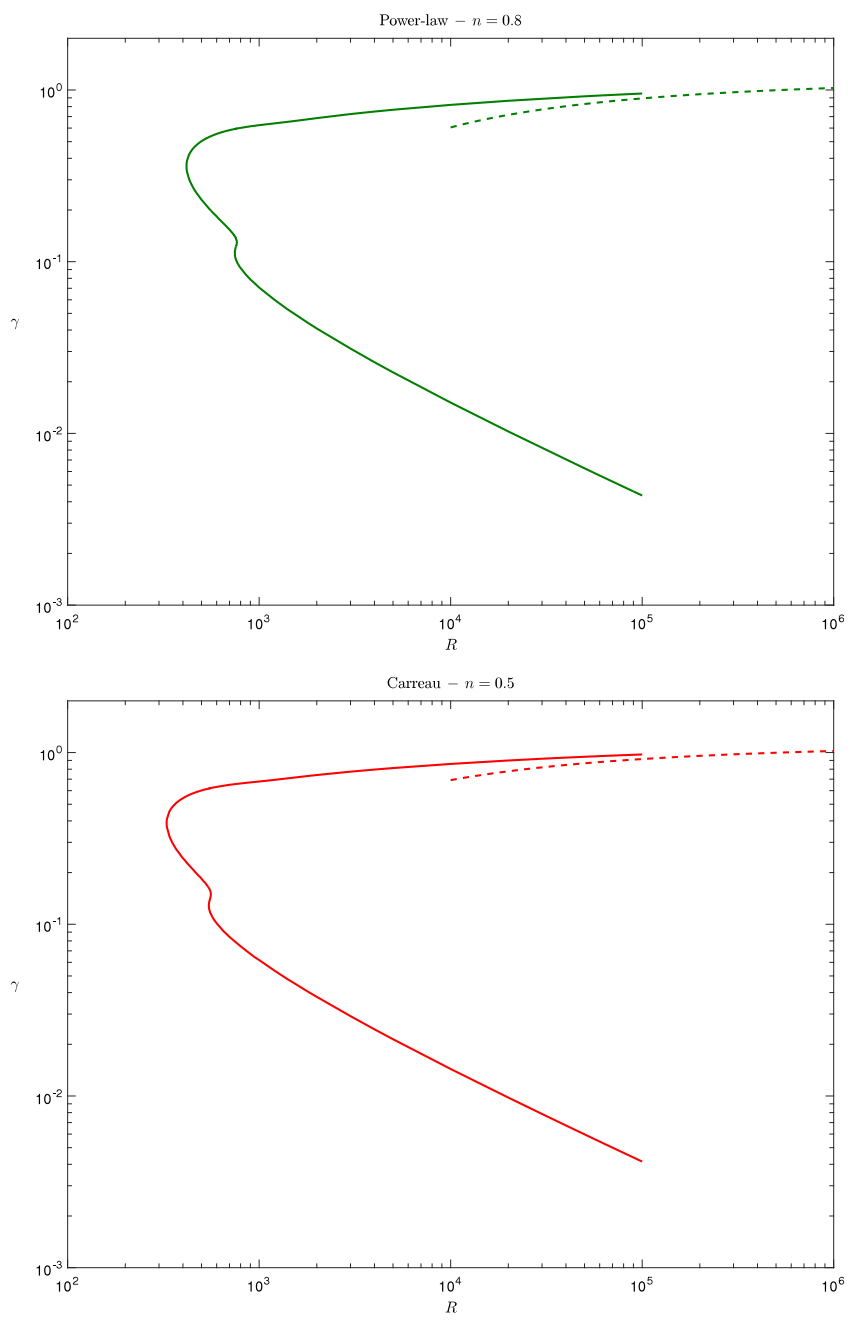

Fig. 2. Neutral stability curves for large $R$ with corresponding asymptotic predictions. Excellent agreement is observed in both cases for every $n$ in the range of interest.

the type I mode appears not to be. In this study we choose to report the results owing from our transformed variables method.

\section{Discussion \& conclusions}

Our results, both asymptotic and numerical, predict differing behaviour for shear-thinning fluids described by the power-law model when compared to those described by the Carreau fluid model. We observe from Fig. 1, where we plot the wavenumber

$\gamma=\sqrt{R^{q-1} \alpha^{2}+\frac{\beta^{2}}{R^{2}}}=\gamma_{A}$,

against the Reynolds number $R$, that the power-law model predicts a strong stabilising effect as the fluid index is decreased, whereas the Carreau model suggests that shear-thinning destabilises the boundary-layer flow. Under the power-law regime the value of both the type I and type II critical Reynolds numbers significantly increases with decreasing $n$ (see Table 2). In addition to this the area encompassed by the neutral curves noticeably shrinks as the effect of shear-thinning increases. Contrary to this, using the Carreau fluid model, we predict that both the critical Reynolds numbers will be marginally decreased as the shear-thinning parameter is increased (see Table 3), and moreover the area encompassed by the neutral curve also significantly increases. In this sense we observe that the type I mode is being strongly destabilised. 
Table 2

The values of the type I and type II critical Reynolds numbers $\left(R_{c}\right)$ for shear-thinning power-law flows as noted by Griffiths et al. [15].

\begin{tabular}{lll}
\hline$n$ & $R_{c}$ (type I) & $R_{c}$ (type II) \\
\hline 0.6 & 703.77 & 1402.0 \\
0.7 & 534.55 & 1006.3 \\
0.8 & 417.49 & 746.23 \\
0.9 & 334.01 & 569.27 \\
\hline
\end{tabular}

Table 3

The values of the type I and type II critical Reynolds numbers $\left(R_{c}\right)$ for a range of shear-thinning Carreau fluid flows.

\begin{tabular}{lll}
\hline$n$ & $R_{c}$ (type I) & $R_{c}$ (type II) \\
\hline 0.25 & 301.11 & 492.11 \\
0.5 & 328.80 & 546.03 \\
0.75 & 364.51 & 612.90 \\
0.95 & 383.93 & 633.34 \\
\hline
\end{tabular}

Fig. 2 outlines the excellent agreement obtained between the results of the asymptotic analysis when compared to our numerical solutions. The large Reynolds number asymptotic analysis does indeed predict boundary-layer stabilisation in the power-law limit and destabilisation in the Carreau limit. This is wholly consistent with the numerical predictions at much lower Reynolds numbers.

In Fig. 3 we plot the spatial branches of the type I mode through the convectively unstable region in order to visualise the growth rates. We denote $-\bar{\alpha}_{\mathrm{i}}=-R^{(q-1) / 2} \alpha_{\mathrm{i}}$ as the spatial growth rate. In all cases we find that the type II growth rates are comparatively very small, this suggests that the type I mode is dominant for all shear-thinning rotating disk boundary-layer flows. Under the power-law framework we find that the type I growth rates are significantly reduced as $n$ decreases whereas results owing from the Carreau fluid model suggest that shear-thinning serves to markedly increase the spatial growth rates.

The opposing results for our two shear-thinning models are indeed quite striking. These surprising results are attributed to the inability of the power-law model to describe shear-thinning flows for vanishing shear-rates, i.e. far from the disk surface. It is well known that for shear-thinning power-law fluids $\mu^{*}\left(\dot{\gamma}^{*} \rightarrow 0\right) \rightarrow$ $\infty$, which is exemplified by our base flow solutions where we see that the viscosity function grows without bound as we move away from the surface of the disk, or equivalently move towards a region of low shear-rate, see Fig. 4. This essentially has the effect of increasing the predicted boundary-layer thickness. Contrastingly, we have that $\mu^{*}\left(\dot{\gamma}^{*} \rightarrow 0\right) \rightarrow \mu_{0}^{*}$ for shear-thinning Carreau fluids. Thus, far from the disk, in regions of low shear-rate, we find that $\mu \rightarrow 1+c_{0}$, and in this case the boundary-layer thickness is reduced. It is this failing of the power-law model in regions of low shear rates that dramatically affects the predicted velocity profiles and therefore our linear stability results.

It transpires that the inability of the power-law model to accurately describe shear-thinning flows in the limit as $\dot{\gamma}^{*} \rightarrow 0$ has such a significant effect on the base flow profiles that it in turn effects the predicted stability characteristics. Therefore it is with relative confidence that we can say that the results owing from the Carreau fluid model do indeed provide a much better description of the observed cross-flow instabilities. Indeed, a recent report by Griffiths et al. [21] shows that shear-thinning strongly destabilises the boundary-layer flow over a flat plate. The results from this study would also suggest that it is the effect of shear-thinning the destabilises the boundary-layer flow over a rotating disk.

These results clearly show the importance of accurately modelling the variation of viscosity within the boundary-layer. The power-law model may be useful for describing experimental results in regions of moderate shear-rate, however, it fails within the confines of our theoretical framework. As such, we conclude
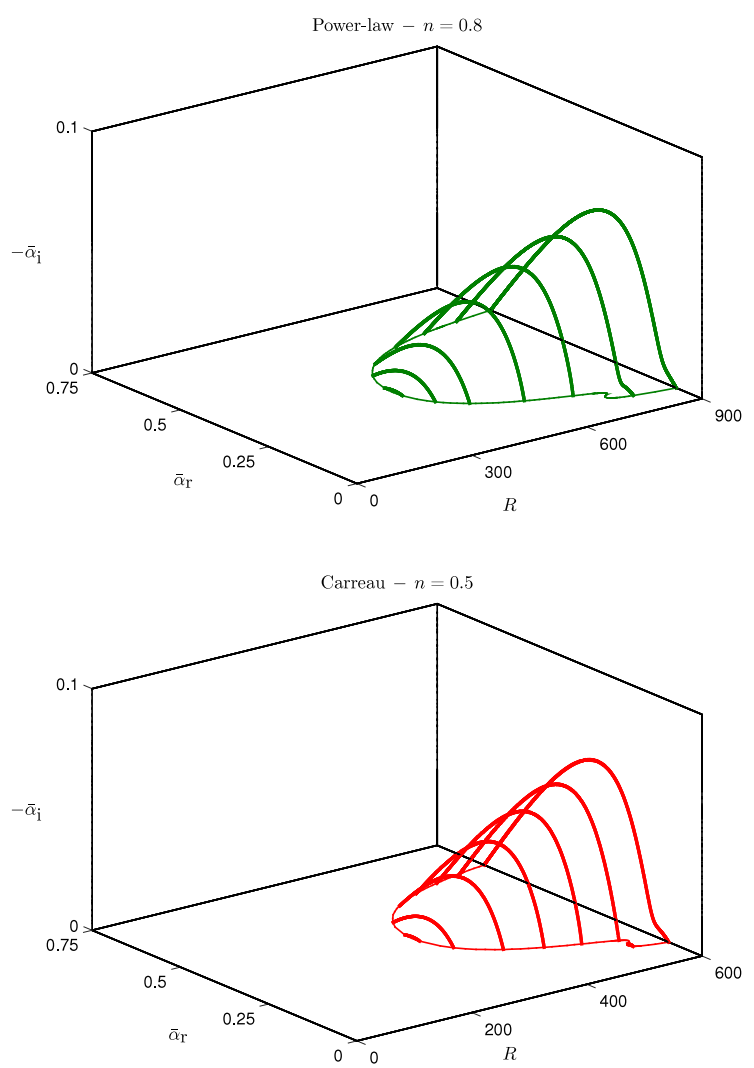

Fig. 3. Linear convective growth rates for stationary mode disturbances of type I through the convectively unstable region for shear-thinning flows. Under the Carreau model the maximum predicted spatial growth rate is larger at $n=0.5$ than it is when compared to the equivalent power-law result with $n=0.8$.

that the Carreau model provides a better physical representation of the boundary-layer flow and hence that the introduction of shearthinning fluids will have a destabilising effect. We should note that similar qualitative results are obtained from the Carreau model if one chooses to make the viscosity function dimensionless with respect to $\mu_{0}^{*}$, as opposed to $\mu_{\infty}^{*}$.

This work clearly motivates the need for detailed experimental results with which to compare our theoretical analysis. To the best of the authors' knowledge no such experiments have yet taken place. Certainly in the Newtonian regime a wealth of literature exists, and this is currently a topic of particular interest, see Imayama et al. [22-24], for example. Personal communication with these authors has revealed the difficulty of obtaining consistently accurate experimental results; therefore we can only envisage that the introduction of non-Newtonian fluids would serve to significantly complicate any experimental procedure.

It is acknowledged that the parallel-flow approximation utilised within the numerical stability analysis means that the perturbation equations solved here are not rigorous at $\mathcal{O}\left(R^{-1}\right)$. Although it is clear that the approximation will lead to inaccuracies at the predicted critical Reynolds numbers, it is our opinion that these will be small. The excellent agreement obtained between our numerical neutral curves and our asymptotic predictions shows that the effects of this approximation are negligible at high Reynolds number.

\section{Acknowledgements}

Part of this work was carried out whilst PTG was receiving monetary support from the Engineering and Physical Sciences Research Council UK. This is gratefully acknowledged. SJG is supported by a Senior Fellowship of the Royal Academy of Engineering and the Leverhulme Trust (LSRF1415/11/29). 

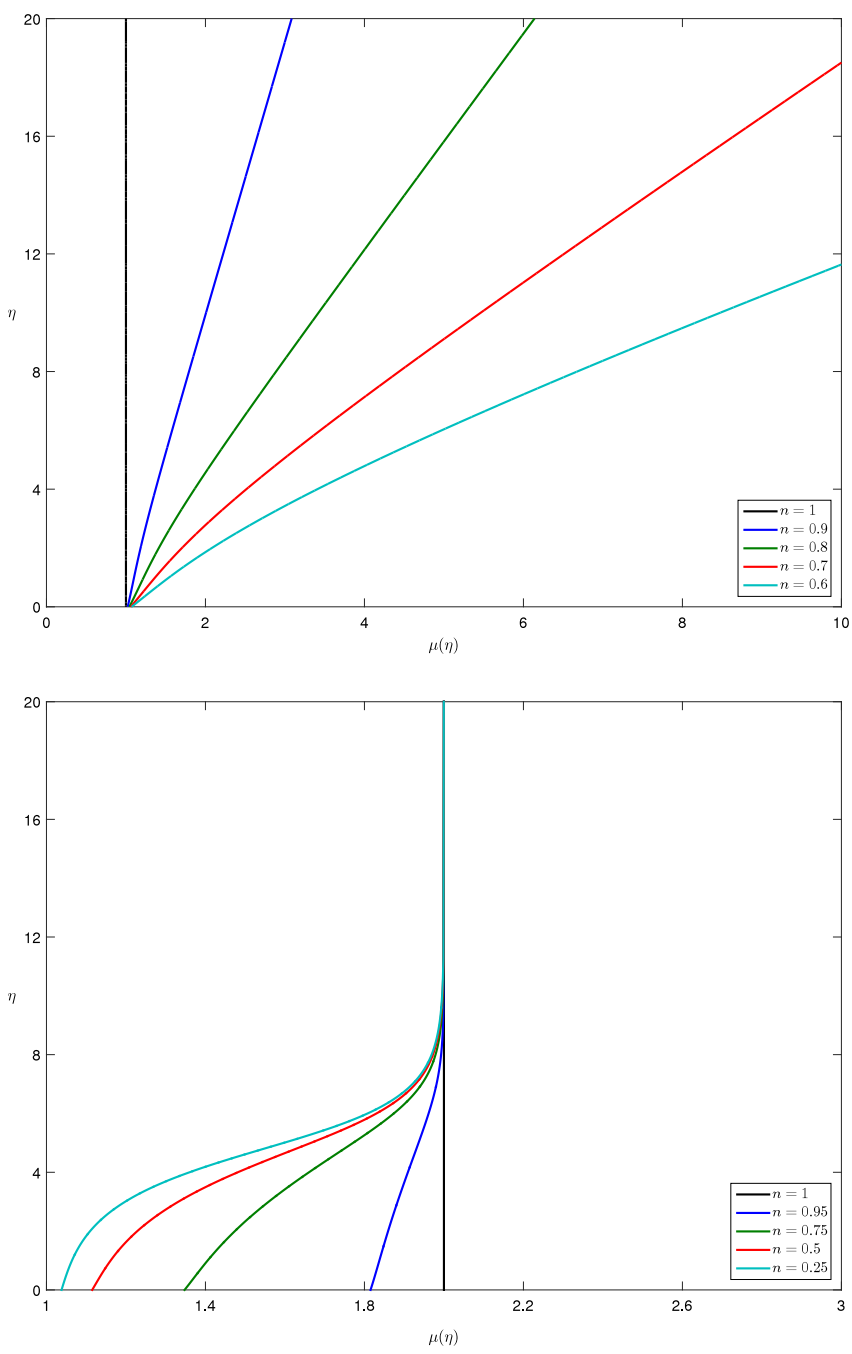

Fig. 4. Base flow viscosity profiles for shear-thinning power-law and Carreau boundary-layer flows. The viscosity functions are defined in (3d) and (3e), respectively.

\section{References}

[1] T. von Kármán, Über laminare und turbulente Reibung, Z. Angew. Math. Mech. 1 (1921) 233-252.
[2] G.K. Batchelor, Note on the class of solutions of the Navier-Stokes equations representing steady non-rotationally symmetric flow, Q. J. Mech. Appl. Math. 4 (1951) 29-41.

[3] U.T. Bödewadt, Die Drehströmung über festem Grund, Z. Angew. Math. Mech. 20 (1940) 241-252.

[4] P.J.Zandbergen, D. Dijkstra, Von Kármán swirling flows, Annu. Rev. Fluid Mech. 19 (1987) 465-491.

[5] N. Gregory, J.T. Stuart, W.S. Walker, On the stability of three-dimensional boundary layers with applications to the flow due to a rotating disk, Philos. Trans. R. Soc. Lond. A Math. Phys. Eng. Sci. 248 (1955) 155-199.

[6] M.R. Malik, The neutral curve for stationary disturbances in rotating-disk flow, J. Fluid Mech. 164 (1986) 275-287.

[7] R.J. Lingwood, Absolute instability of the boundary layer on a rotating disk, J. Fluid Mech. 299 (1995) 17-33.

[8] P. Hall, An asymptotic investigation of the stationary modes of instability of the boundary layer on a rotating disc, Proc. R. Soc. Lond. Ser. A Math. Phys. Eng. Sci. 406 (1986) 93-106.

[9] S.J. Garrett, N. Peake, The stability and transition of the boundary layer on a rotating sphere, J. Fluid Mech. 456 (2002) 199-218.

[10] S.J. Garrett, Z. Hussain, S.O. Stephen, The cross-flow instability of the boundary layer on a rotating cone, J. Fluid Mech. 422 (2009) 209-232.

[11] Z. Hussain, S.J. Garrett, S.O. Stephen, P.T. Griffiths, The centrifugal instability of the boundary-layer flow over a slender rotating cone in an enforced axial free strem, J. Fluid Mech. 788 (2016) 70-94.

[12] R.J. Lingwood, P.H. Alfredsson, Instabilities of the von Kármán boundary layer, Appl. Mech. Rev. 67 (3) (2015) 030803.

[13] P.T. Griffiths, Flow of a generalised Newtonian fluid due to a rotating disk, J. Non-Newton. Fluid Mech. 221 (2015) 9-17.

[14] P.P. Dabrowski, Boundary-layer Flows in non-Newtonian Fluids (Ph.D thesis), School of Mathematical Sciences, The University of Adelaide, 2004.

[15] P.T. Griffiths, S.O. Stephen, A.P. Bassom, S.J. Garrett, Stability of the boundary layer on a rotating disk for power law fluids, J. Non-Newtonian Fluid Mech. 207 (2014) 1-6.

[16] P.T. Griffiths, Hydrodynamic Stability of Non-Newtonian Rotating BoundaryLayer Flows (Ph.D thesis), School of Mathematics, University of Birmingham, 2015.

[17] P.T. Griffiths, S.J. Garrett, S.O. Stephen, The neutral curve for stationary disturbances in rotating disk flow for power-law fluids, J. Non-Newtonian Fluid Mech. 213 (2014) 73-81.

[18] S.J. Garrett, N. Peake, The stability of the boundary layer on a rotating sphere in a uniform axial flow, Eur. J. Mech. B 23 (2004) 241-253.

[19] B. Alveroglu, A. Segalini, S.J. Garrett, The effect of surface roughness on the convective instability of the bek family of boundary-layer flows, Eur. J. Mech. B 56 (2016) 178-187.

[20] S.J. Garrett, A.J. Cooper, J.H. Harris, M. Özkan, A. Segalini, P.J. Thomas, On the stability of von Kármán rotating-disk boundary layers with radial anisotropic surface roughness, Phys. Fluids 28 (2016) 014104.

[21] P.T. Griffiths, M.T. Gallagher, S.O. Stephen, The effect of non-Newtonian viscosity on the stability of the Blasius boundary layer, Phys. Fluids 28 (2016) 074107.

[22] S. Imayama, P.H. Alfredsson, R.J. Lingwood, A new way to describe the transition characteristics of a rotating-disk boundary-layer flow, Phys. Fluids 24 (2012) 031701

[23] S. Imayama, P.H. Alfredsson, R.J. Lingwood, An experimental study of edge effect on rotating-disk transition, J. Fluid Mech. 716 (2013) 638-657.

[24] S. Imayama, P.H. Alfredsson, R.J. Lingwood, On the laminar-turbulent transition of the rotating-disk flow: the role of absolute instability, J. Fluid Mech. 745 (2014) 132-163. 